\title{
A System for the Fast Prototyping of Multidimensional Image Retrieval
}

\author{
Carlo Meghini, Fabrizio Sebastiani and Umberto Straccia
}

\begin{abstract}
Multidimensional image retrieval (MIR) views an image as a multidimensional object, where each dimension is a channel for retrieval. MIR has the potential of putting at work together the many methods and techniques for image retrieval proposed in several different fields of computer science. We have developed a model of MIR, based on a fuzzy description logic, that identifies two main dimensions in an image (form and content) and views MIR as a special form of uncertain implication. In this paper, we present ARIANNA, a system that implements the model. ARIANNA allows to quickly develop a prototype of a MIR application, and use it to test the adequacy of the application to the user's functional requirements.
\end{abstract}

Keywords: Multimedia databases, Content-based indexing/retrieval

\footnotetext{
${ }^{*}$ Corresponding author. Surface mail address: Consiglio Nazionale delle Ricerche, Istituto di Elaborazione dell'Informazione. Via S. Maria 46 - 56126 Pisa, Italy. E-mail address: meghini@iei.pi.cnr.it
} 


\section{Introduction}

Research on image retrieval (IR) has witnessed a booming interest during the last decade. The most striking feature of this research is its simultaneous but independent materialization within several fields of computer science. This fact reveals that there are many different aspects involved in IR, each requiring a specific background and methodology to be successfully tackled; but also that there may be complementary approaches to the same problems, not only within the same discipline (such as two different index structures for image data), but also cutting across different disciplines (such as similarity- versus semantic-based image retrieval). Such a richness of paradigms, methods and systems, on the long run, may result in a fragmentation prone to slow down progress. In order to overcome this problem, we have developed a model of IR [MSS97b], named the Terminological Image Retrieval Model (TIM) after the tool we have used to specify it, that places in a unified and coherent perspective the many efforts and results that are being produced under the IR label. The model has been successively extended to deal with multimedia [MSS97a]. The basic feature of TIM is to allow the capturing of all kinds of retrieval on images that have been deemed as useful, and therefore investigated in the various areas mentioned above. These kinds of retrieval can be broadly classified on the basis of the aspect of images that each of them addresses. Thus we have a first, broad categorization of IR in: syntactic similarity vs semantic IR. The former, in turn, can be categorized depending on the addressed image feature: thus we have color-, shape-, texture-based similarity, and there may be more.

TIM is parametric with respect to the functions that are used to asses similarity of image features, as it is well-known that those functions vary depending on the subject matter and the goals underlying the MIR application being developed.

The present paper describes an implementation of TIM aimed at supporting the construction of fast prototypes of MIR applications. For space reasons, the presentation just touches upon the most important aspects of ARIANNA. The readers interested in knowing more about the model or the system are referred to a long version of this paper [MSS98].

\section{The terminological image retrieval model}

As any retrieval model, TIM consists of a language for representing images, a language for representing queries, and a function, called retrieval function, establishing the retrieval status value of each image for each query. RSV estimates the degree of relevance of an image to a query. The language used by TIM for image and query representation is a fuzzy description logic [Str98], while the retrieval function is based on the implication relation of the same logic.

\subsection{Image representation}

In TIM, each image has a form and a content dimension. The former, named "image layout", consists of the pixels that make up the image. No language facility is provided to represent a layout, as it can be dealt with in an entirely automatic way. Content, on the other hand, requires interpretation to be disclosed and is therefore of a subjective nature. TIM provides the means to represent explicitly image contents in terms of the assertions of the fuzzy description logic $\mathcal{A L C}$.

The syntactic components of $\mathcal{A L C}$ are: individuals (names for objects), concepts (unary predicate symbols) and roles (binary predicate symbols). Concepts can be either primitive (just names) or complex, i.e. expressions involving primitive concepts and constructors, which, not surprisingly, closely mimic Boolean operators. For instance, the complex concept Musician $\square \forall P l a y s$. $\neg$ Electriclnstrument is obtained by combining the primitive concepts Musician and Electriclnstrument and the primitive 


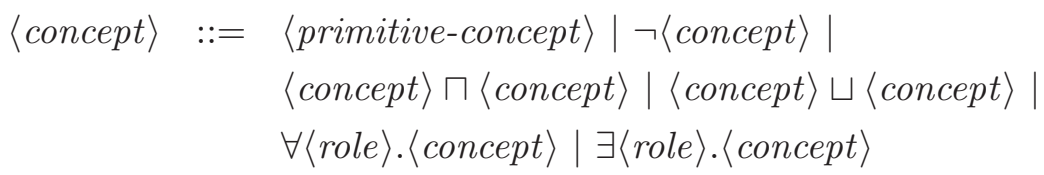

Figure 1: Grammar rules for $\mathcal{A L C}$ concepts

role Plays by the conjunction $(\sqcap)$, universal quantification $(\forall)$ and negation $(\neg)$ constructors. Under the intended interpretation, such concept denotes the musicians who do not play any electric instrument. The syntax rule for $\mathcal{A L C}$-concepts are given in Figure 1.

A fuzzy assertion is an expression of the form $\langle\alpha, n\rangle$, where $n \in[0,1]$ and $\alpha$ is one of the following: (1) $C(a)$, asserting that the individual $a$ is an instance of the concept $C$; for example, (Musician $\sqcap$ Teacher)(tim) makes the individual constant tim a Musician and a Teacher; $(2) R\left(a_{1}, a_{2}\right)$ (e.g. Friend(tim,tom)); (3) $C_{1} \sqsubseteq C_{2}$, asserting that $C_{1}$ is more specific than $C_{2}$ (e.g. Pianist $\sqsubseteq$ (Artist $\sqcap \exists$ Plays.Piano)). A set of assertions will be called a knowledge base.

Semantically, concepts and roles are interpreted as fuzzy sets. A fuzzy interpretation is a pair $\mathcal{I}=\left(\Delta^{\mathcal{I}},{ }^{\mathcal{I}}\right)$, where $\Delta^{\mathcal{I}}$ is the domain, whereas ${ }^{\mathcal{I}}$ is an interpretation function mapping: (a) different individuals to different elements of $\Delta^{\mathcal{I}}$, (b) each concept into a membership degree function $\Delta^{\mathcal{I}} \rightarrow[0,1]$, and (c) each role into a membership degree function $\Delta^{\mathcal{I}} \times \Delta^{\mathcal{I}} \rightarrow[0,1]$. In order to reflect intuition, ${ }^{\mathcal{I}}$ has to satisfy the constraints imposed by the meaning of the operators in the context of fuzzy set theory; thus, for instance (for all $\left.d \in \Delta^{\mathcal{I}}\right),\left(C_{1} \sqcap C_{2}\right)^{\mathcal{I}}(d)$ must be $\min \left\{C_{1}{ }^{\mathcal{I}}(d), C_{2}^{\mathcal{I}}(d)\right\}$, while $(\forall R . C)^{\mathcal{I}}(d)$ must be $\min _{d^{\prime} \in \Delta^{\mathcal{I}}}\left\{\max \left\{1-R^{\mathcal{I}}\left(d, d^{\prime}\right), C^{\mathcal{I}}\left(d^{\prime}\right)\right\}\right\}$.

An interpretation $\mathcal{I}$ satisfies a fuzzy assertion $\langle C(a), n\rangle$ (resp. $\langle R(a, b), n\rangle$ and $\left\langle C_{1} \sqsubseteq C_{2}, n\right\rangle$ ) iff $C^{\mathcal{I}}\left(a^{\mathcal{I}}\right) \geq n\left(\operatorname{resp} . R^{\mathcal{I}}\left(a^{\mathcal{I}}, b^{\mathcal{I}}\right) \geq n\right.$ and $\left.\min _{d \in \Delta^{\mathcal{I}}}\left\{\max \left\{1-C_{1}{ }^{\mathcal{I}}(d), C_{2}{ }^{\mathcal{I}}(d)\right\}\right\} \geq n\right)$. An interpretation $\mathcal{I}$ satisfies (is a model of) a fuzzy KB $\Sigma$ iff $\mathcal{I}$ satisfies each element of $\Sigma$. A fuzzy KB $\Sigma$ entails a fuzzy assertion $\gamma$ (written $\Sigma \models \gamma$ ) iff every model of $\Sigma$ also satisfies $\gamma$. Given a fuzzy KB $\Sigma$ and a fuzzy assertion $\alpha$, we define the maximal degree of truth of $\alpha$ with respect to $\Sigma$ (written Maxdeg $(\Sigma, \alpha))$ to be $\max \{n>0: \Sigma \models\langle\alpha, n\rangle\}(\max \emptyset=0)$.

Let us now see how the fuzzy description logic is to be employed in order to represent image contents. Let $l$ be an image layout uniquely identified by the individual I. In TIM, $l$ may have an arbitrary number of associated content descriptions. Each such content description is a set of fuzzy assertions, given by the union of four component subsets: (1) The layout identification, a singleton with a fuzzy assertion of the form $\langle\operatorname{Self}(I), 1\rangle$ whose role is to associate a content description with the layout it refers to. The layout identification is the same for all content descriptions of the same layout. $\sigma(\mathrm{I})$ denotes the set of the content descriptions associated to the layout $l$. (2) The object anchoring, a set of fuzzy assertions of the form $\langle\operatorname{Rep}(r, o), n\rangle$ where $r$ is an individual that uniquely identifies a region $r$ of $l$ and $o$ is an individual that uniquely identifies the object represented in the region $r$. (3) The situation anchoring, a set of fuzzy assertions of the form $\langle\operatorname{About}(\mathrm{I}, \mathrm{o}), n\rangle$ where I and $o$ are as above. By using these assertions, it can be stated what the situation described by the layout is "globally" about. (4) The situation description, a set of fuzzy simple assertions (where none of the symbols Self, Rep or About occur), describing important facts stated in the layout about the individuals identified by assertions of the previous two kinds.

\subsection{Query representation}

Queries referring to the form dimension of images are called visual queries. TIM addresses the following visual query types: (a) concrete visual queries: these consist of full-fledged images that 


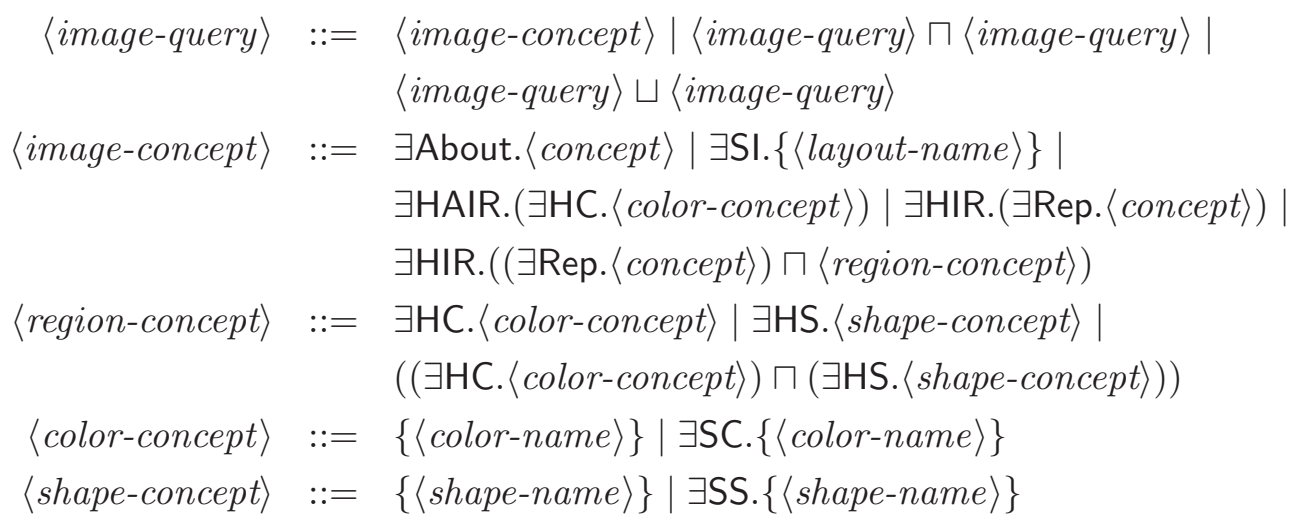

Figure 2: Grammar rules for image queries

are submitted to the system as a way to indicate a request to retrieve "similar" images; the addressed aspect of similarity may concern color, texture, appearance, and so on; (b) abstract visual queries: these are artificially constructed image elements (hence, "abstractions" of image layouts) that address specific aspects of image similarity; they can be further categorized into: (b.1) color queries: specifications of color patches, used to indicate a request to retrieve those images in which a similar color patch occurs; (b.2) shape queries: specifications of one or more shapes (closed simple curves in the 2D space), used to indicate a request to retrieve those images in which the specified shapes occur as contours of significant objects; (b.3) any combination of the above.

In order to express these queries, TIM offers a language that is considerably richer than the language for representing images, as it includes the symbols (termed SPSs) for expressing all the above query kinds. Essentially, SPSs are roles that make up an image mereology. For TIM, the atomic image element is the atomic region, that is a connected region which is homogeneous from the color viewpoint. Connected aggregates of atomic regions constitute regions. Colors and shapes are associated to regions of any kind. We have therefore the following SPSs:

- HAIR(i,r) relates the image layout $i$ to one of its atomic regions $r$;

- $H I R(i, r)$ relates the image layout $i$ to one of its regions $r$;

- $\mathrm{HS}(r, s)$ relates an image region $r$ to its shape $s$;

- $\mathrm{HC}(r, c)$ relates an image region $r$ to its color $c$.

In order to properly handles SPSs, we restrict the models of TIM image databases to those that capture the intended meaning of these symbols, expressed through conditions that will not be given here for space reasons.

The syntax of image queries is given in Figure 2. The first thing to observe is the presence, in the clauses defining image-, color- and shape-concepts, of a new concept constructor of the form $\{a\}$ where $a$ is an individual, which may be a layout-, a color- or a shape-name, respectively. This constructor is called singleton, and represents a concept having only the individual $a$ as instance. Image queries are thus concepts of the DL $\mathcal{A L C O}$, which extends $\mathcal{A L C}$ with the singleton constructor. It has been proved that the additional expressive power of $\mathcal{A L C O}$ over $\mathcal{A L C}$ has no impact on the complexity of the image retrieval problem. 
An image query is a combination (through $\sqcap$ and $\sqcup$ ) of image-concepts, each of which may have one of four forms, illustrated in the following in the same order as they appear in Figure 2.

First, an image-concept may be a query on some content object, explicitly asserted to be related to the sought images through an About role assertion (termed "situation anchoring"). In the query, the object in question is required to be an instance of concept, that is an $\mathcal{A L C O}$ concept built with the symbols used for situation descriptions. For instance, under the obvious lexicon, the images about an Italian musician are retrieved via the query $\exists$ About.(Musician $\sqcap \exists$ Born.Italy). Second, an image-concept may be a concrete visual query. In this case, a prototype image layout $l$ is to be provided with the query; this is done by specifying the singleton with the layout name I in the scope of the existential quantification on the SPS SI. By so doing, the similarity with $l$ is captured in the query. Third, an image-concept may be a color abstract visual query, expressed via an existential quantification on the HC SPS, followed by a color-concept; the latter is a singleton with the name of the color, optionally preceded by a color similarity predicate. Finally, an image-concept may be a query on an image region. This kind of queries come in two forms:

1. The first form is meant to address the content dimension, and just consists of a Rep clause. In order to qualify for this kind of queries, an image must have an associated content description containing a Rep role assertion (object anchoring), relating a region of the image to an individual constant that is an instance of the concept that follows.

2. The second form extends the first with an additional condition (region-concept) on the color or the shape (or both) of the involved region. A shape condition is expressed via a shape-concept, which is strictly analogous to color-concept.

As an instance of an image query, let us consider the query asking for the images showing a cylindric reddish hat. This query can be expressed by the following image concept:

$$
\exists \text { HIR. }((\exists \text { Rep.Hat }) \sqcap(\exists \text { HC. } \exists \text { SC. }\{\text { red }\}) \sqcap(\exists \text { HS. }\{\text { cylinder }\}))
$$

The above query presents an interesting case of mixed form- and content-based image retrieval. In particular, the Rep clause refers to the semantics of the image, namely to what an object is. An image is retrieved only if it displays something that has been explicitly asserted to be a hat. The $\mathrm{HC}$ clause refers to image form, and requires, in the retrieved images, the presence of a patch of color similar to red. The HS clause poses a condition on the contour of an atomic image region. The conjunction of these three clauses constraints the condition that they each of them expresses to be true of the same region, thus capturing the query spelled out above.

\subsection{The retrieval function}

A TIM image database has three main components: a collection of image layouts; a collection of content descriptions associated to these layouts; and a knowledge base, providing definitions of the concepts employed in content representations, as well as general knowledge on the domain of discourse. More precisely, an image database is a triple $D B=\left\langle D, \Sigma_{C}, \Sigma_{D}\right\rangle$ where $D$ is a set of layouts, $\Sigma_{C}$ is the set of content descriptions of the layouts in $D$, and $\Sigma_{D}$ is a set of fuzzy assertions.

In response to a query $Q$ addressed to a document base $D B=\left\langle D, \Sigma_{C}, \Sigma_{D}\right\rangle$, each image is attributed a retrieval status value $m$ given by:

$$
m=\max _{\delta \in \sigma(\mathrm{i})}\left\{\overline{\operatorname{Maxdeg}}\left(\Sigma_{D} \cup \delta, Q(\mathrm{i})\right)\right\} .
$$

where $\overline{M a x d e g}$ is the restriction of Maxdeg that assigns to SPSs the intended semantics. 
As an example, let us consider an image database containing just the image named i, whose $\Sigma_{C}$ component consists of the content description $\{\langle\operatorname{Self}(\mathrm{i}), 1\rangle,\langle\operatorname{About}(\mathrm{i}, \mathrm{o}), .8\rangle,\langle$ DonGiovanni(o), 1$\rangle\}$, and whose $\Sigma_{D}$ component consists of the assertions: $\langle$ DonGiovanni $\sqsubseteq$ EuropeanOpera, 1$\rangle$,

$\langle$ EuropeanOpera $\sqsubseteq$ Opera $\sqcap$ ( $\exists$ ConductedBy.European $), .9\rangle$. Suppose we are interested in documents containing images about operas conducted by a European director. To this end, we can use the query $\exists$ About.(Opera $\sqcap \exists$ ConductedBy.European). It can be verified that the RSV attributed to $\mathrm{i}$ is .8 .

\section{The decomposition strategy}

The logical kernel of the model implementation is the query evaluation procedure (QEP, for short), which, given a query $Q$ and an image database $D B$, returns a ranking of the images in $D B$. The basic tasks of the QEP are (1) the identification of the structure sub-queries that are embedded in the query; (2) the evaluation of the identified sub-queries; (3) the calculation of the RSV of the image on the basis of the evaluated sub-queries. The QEP relies on special processors for the evaluation of sub-queries, and on a fuzzy $\mathcal{A L C O}$ theorem prover (TP, for short) for the evaluation of semantic sub-queries as well as for the calculation of the overall document RSV. For space reasons, we cannot discuss the TP here. The interested reader is referred to [Str98 $]^{1}$. The QEP follows a method, called the decomposition strategy, which guarantees the correctness of the result, given the correctness of the sub-query evaluations. The rest of the section is devoted to the illustration of the decomposition strategy.

\subsection{Foundations}

Let $Q$ be a generic query and i a candidate image in $D B$. As established by the model, the RSV of i to $Q$ is the value $m$ associated to the crisp assertion $Q(\mathrm{i})$. In order to derive $m$, the function $\Phi$ is applied to $Q(\mathrm{i})$, thus obtaining the decomposition of query $Q$, i.e. a set of fuzzy assertions $\Phi(Q(\mathrm{i}))$ which satisfies the following property: For each $\delta \in \sigma(\mathrm{i})$ :

$$
\overline{\operatorname{Maxdeg}}\left(\Sigma_{D} \cup \delta, Q(\mathrm{i})\right)=\operatorname{Maxdeg}\left(\Sigma_{D} \cup \Phi(Q(\mathrm{~d})) \cup \delta, Q(\mathrm{i})\right) .
$$

From this, the decomposition principle that is at the basis of the implementation of the model follows:

Query Decomposition Principle. The RSV of the image i to the query $Q$ is given by the maximal degree of truth of $Q(\mathrm{i})$ with respect to the knowledge base consisting of $\Phi(Q(\mathrm{~d}))$ and $\Sigma_{D}$, maximized over the set of content descriptions $\sigma(\mathrm{i})$.

A formal proof of the correctness and completeness of the query decomposition principle can be found in a longer version of this paper [MSS98]. Operatively, this principle permits to perform query evaluation in the general context of fuzzy $\mathcal{A L C O}$, once the sub-queries involving the special symbols SPSs are identified and evaluated by the function $\Phi$ and the corresponding fuzzy assertions are collected in $\Phi(Q(\mathrm{~d}))$.

Now let us see how $\Phi$ must be defined in order to license the query decomposition principle. For greater clarity, being a function defined on the query language, $\Phi$ will be introduced by following the query language syntactical structure.

\footnotetext{
${ }^{1}$ To download the TP: http://faure.iei.pi.cnr.it/ straccia/html/software/software.html.
} 


\begin{tabular}{|c|c|}
\hline$x$ & $\Phi(x)$ \\
\hline$(C \sqcap D)(\mathrm{i}) \quad(C \sqcup D)(\mathrm{i})$ & $\Phi(C(\mathrm{i})) \cup \Phi(D(\mathrm{i}))$ \\
\hline$(\exists S I .\{q i\})(i)$ & $\left\{\left\langle\mathrm{SI}(\mathrm{i}, \mathrm{qi}), \sigma_{i}\left(\mathrm{i}^{\mathcal{I}}, \mathrm{qi}^{\mathcal{I}}\right)\right\rangle\right\}$ if $\left.\left.\sigma_{i}\left(\mathrm{i}^{\mathcal{I}}, \mathrm{qi}^{\mathcal{I}}\right)\right\rangle\right\}>0, \emptyset$ otherwise \\
\hline$(\exists$ About. $C)(\mathrm{i})$ & $\emptyset$ \\
\hline$(\exists$ HAIR. $\exists$ HC. $\{c\})(i)$ & $\{\langle x, 1\rangle\}$ if $\mathrm{i}^{\mathcal{I}}$ has an atomic region of color $\mathrm{c}^{\mathcal{I}}, \emptyset$ otherwise \\
\hline$(\exists$ HAIR. $\exists$ HC. $\exists S C .\{c\})(i)$ & $\{\langle x, n\rangle\}$ if $n$, the best approximation of $\mathrm{c}^{\mathcal{I}}$ in $\mathrm{i}^{\mathcal{I}}$, is positive; $\emptyset$ otherwise \\
\hline$(\exists \mathrm{HIR} . C)(\mathrm{i})$ & $\begin{array}{l}\left\{\left\langle\operatorname{HIR}\left(\mathrm{i}, \mathrm{r}_{1}\right), 1\right\rangle, \ldots,\left\langle\operatorname{HIR}\left(\mathrm{i}, \mathrm{r}_{k}\right), 1\right\rangle\right\} \cup \Phi\left(C\left(\mathrm{r}_{1}\right)\right) \cup \ldots \cup \Phi\left(C\left(\mathrm{r}_{k}\right)\right),(k \geq 0), \\
\text { for all regions } \mathrm{r}_{j}{ }^{\mathcal{I}} \text { in } \mathrm{i}^{\mathcal{I}} \text { such that } \operatorname{Rep}\left(\mathrm{r}_{j}, \mathrm{o}\right) \in \delta \text { for some } \delta \in \sigma(\mathrm{i})\end{array}$ \\
\hline$(\exists \operatorname{Rep} . C)(\mathrm{r})$ & $\emptyset$ \\
\hline$((\exists \operatorname{Rep} . C) \sqcap D)(\mathrm{r})$ & $\Phi(D(r))$ \\
\hline$(\exists \mathrm{HC} \cdot\{\mathrm{c}\})(\mathrm{r})$ & $\{\langle\mathrm{HC}(\mathrm{r}, \mathrm{c}), n\rangle\}$ if $n$, the percentage of $\mathrm{c}^{\mathcal{I}}$ in $\mathrm{r}^{\mathcal{I}}$, is positive; $\emptyset$ otherwise \\
\hline$(\exists \mathrm{HC} . \exists S C .\{\mathrm{c}\})(\mathrm{r})$ & $\{\langle x, n\rangle\}$ if $n=\max _{c \in \mathcal{C}} v_{c}$ is positive; $\emptyset$ otherwise \\
\hline$(\exists \mathrm{HS} .\{\mathrm{s}\})(\mathrm{r})$ & $\{\langle\mathrm{HS}(\mathrm{r}, \mathrm{s}), 1\rangle\}$ if $\mathrm{s}^{\mathcal{I}}$ is the shape of $\mathrm{r}^{\mathcal{I}}, \emptyset$ otherwise \\
\hline$(\exists \mathrm{HS} . \exists \mathrm{SS} .\{\mathrm{s}\})(\mathrm{r})$ & $\begin{array}{l}\{\langle x, n\rangle\} \text { if } n, \text { the similarity between the shape of } \mathrm{r}^{\mathcal{I}} \text { and } \mathrm{s}^{\mathcal{I}} \text { is positive; } \\
\emptyset \text { otherwise }\end{array}$ \\
\hline
\end{tabular}

Figure 3: The decomposition function for image queries

\subsection{Decomposition and evaluation of sub-queries}

The decomposition and evaluation of image queries is presented in Figure 3. Recall that the object denoted by the individual o is written as $\circ^{\mathcal{I}}$. Concrete visual queries, having the form $(\exists \mathrm{SI}$. $\{$ qi\})(i) are evaluated by generating the fuzzy assertion stating the similarity between the given image layout $i$ and the query layout qi, with degree of truth equal to the degree of similarity between these layouts, as established by the global similarity function $\sigma_{i}$. Note that in case the latter value is zero, no assertion is generated in order not to block the inference on the rest of the query. The same behavior is adopted whenever a similarity function is involved.

Queries on situation anchoring, formulated in terms of the About SPS, are just ignored by $\Phi$, as the knowledge for their evaluation is already part of the image database, namely it is contained in the content descriptions collected in $\Sigma_{C}$ and in the context knowledge base $\Sigma_{D}$. The same applies to queries on object anchoring, formulated in terms of the Rep SPS. These queries may stand alone (i.e. be of the form $(\exists \operatorname{Rep} . C)(\mathrm{r}))$ or be conjoined to a region concept (i.e. $((\exists \operatorname{Rep} . C) \sqcap D)(\mathrm{r}))$; in both cases, the content sub-query gives no contribution to $\Phi(Q(\mathrm{~d}))$.

Abstract visual queries come in two sorts, depending on the kind of image region addressed. The first and simplest sort address exclusively atomic regions. It consists of color queries and aim at re-

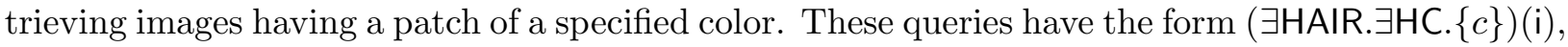
where $\mathrm{c}$ is the name of the color that an atomic region of the image layout named $\mathrm{i}$ must have. If this is indeed the case, $\Phi$ evaluates the query by generating the fuzzy assertion made by attaching to the query assertion with degree of truth 1 . If not, the empty set is generated. Optionally, a similarity condition on the specified color may be stated, yielding queries of the form $(\exists$ HAIR. $\exists$ HC. $\exists S C .\{c\})(i)$. The specification of the color similarity condition radically changes the query evaluation, which yields, as degree of truth, the degree of similarity between the given color and the color of the atomic regions of $i$ that comes closest to it. If $i$ has an atomic region of color 
c, then the degree of truth is $1^{2}$; otherwise, the evaluation produces the "best match" among i's colors and c. As a desirable consequence, the latter type of color queries generalizes the former.

The second sort of abstract visual queries address both atomic and non-atomic regions and takes the general form ( $\exists$ HIR.C)(i). As for the other mereological symbols, $\Phi$ treats these queries by generating an assertion of the form $\langle\mathrm{HIR}(\mathrm{i}, \mathrm{r}), 1\rangle$ for each region $r$ of $i$ which is the subject of an object anchoring assertion, while recursively applying itself to the assertion $C(\mathrm{r})$. The reason for this is that $C$ is bound to include a Rep clause, which, of course, restricts the candidate regions to all and only those referenced by object anchorings. As discussed above, $C$ may optionally contain a region concept, which may be a color query, a shape query or a conjunction of the two. The last case is handled, as customary, by separately evaluating the conjuncts, and is not reported in Figure 3 for brevity. Let us quickly review the first two cases:

- $(\exists \mathrm{HC} .\{c\})(r)$ : is evaluated by generating the corresponding fuzzy assertion, having as degree of truth the percentage of color $c$ in the region $r$.

- $(\exists \mathrm{HC} . \exists S C .\{c\})(r)$ : the truth degree assigned in this case is, for each color $c$ in $r$, the maximum over the values $v_{c}$, where $v_{c}$ is the minimum between the similarity between $c$ and $c^{\mathcal{I}}$, and the percentage of $c$ in $r$. It is easy to verify that this is a generalization over the previous case.

- $(\exists \mathrm{HS} .\{s\})(r)$ : if the shape of $r$ equals $s$, the evaluation of this query yields the corresponding assertion with degree 1; otherwise, no assertion is generated.

- $(\exists$ HS. $\exists S S .\{s\})(r)$ : same as before, except that in this case the similarity between r's shape and $\mathrm{s}$ is assigned as degree of truth to the corresponding assertion.

\section{A prototypical implementation}

The prototype system that we have developed, named ARIANNA, consists of two main modules: the indexing module (hereafter IM for short) supporting the acquisition of images and the creation of the various representations needed for performing retrieval, and the query module (QM), performing query evaluation.

\subsection{The indexing module}

Figure 4 illustrates the various operations comprising image acquisition in what may be considered as the typical sequence (rectangular boxes represent data, while ovals represent modules).

Filtering and Size Reduction Acquisition begins from an Input Image, which may be any image in GIF or JPEG format. The input image is reduced, if necessary, to the system size, which is a parameter currently fixed to $128 \times 128$ pixels. The reduction is performed by means of a resampling technique. After size reduction, the RGB color space is abandoned for the HSB space, and noise reduction is performed on the image, by applying a color median filter. The result is the Basic Image. Figure 5 presents a sample input image (left) and the corresponding basic image.

\footnotetext{
${ }^{2}$ At least as long as $\sigma_{c}\left(c^{\mathcal{I}}, c^{\mathcal{I}}\right)=1$, which would seem a quite reasonable assumption on similarity functions, even though it has not been so stated for generality.
} 


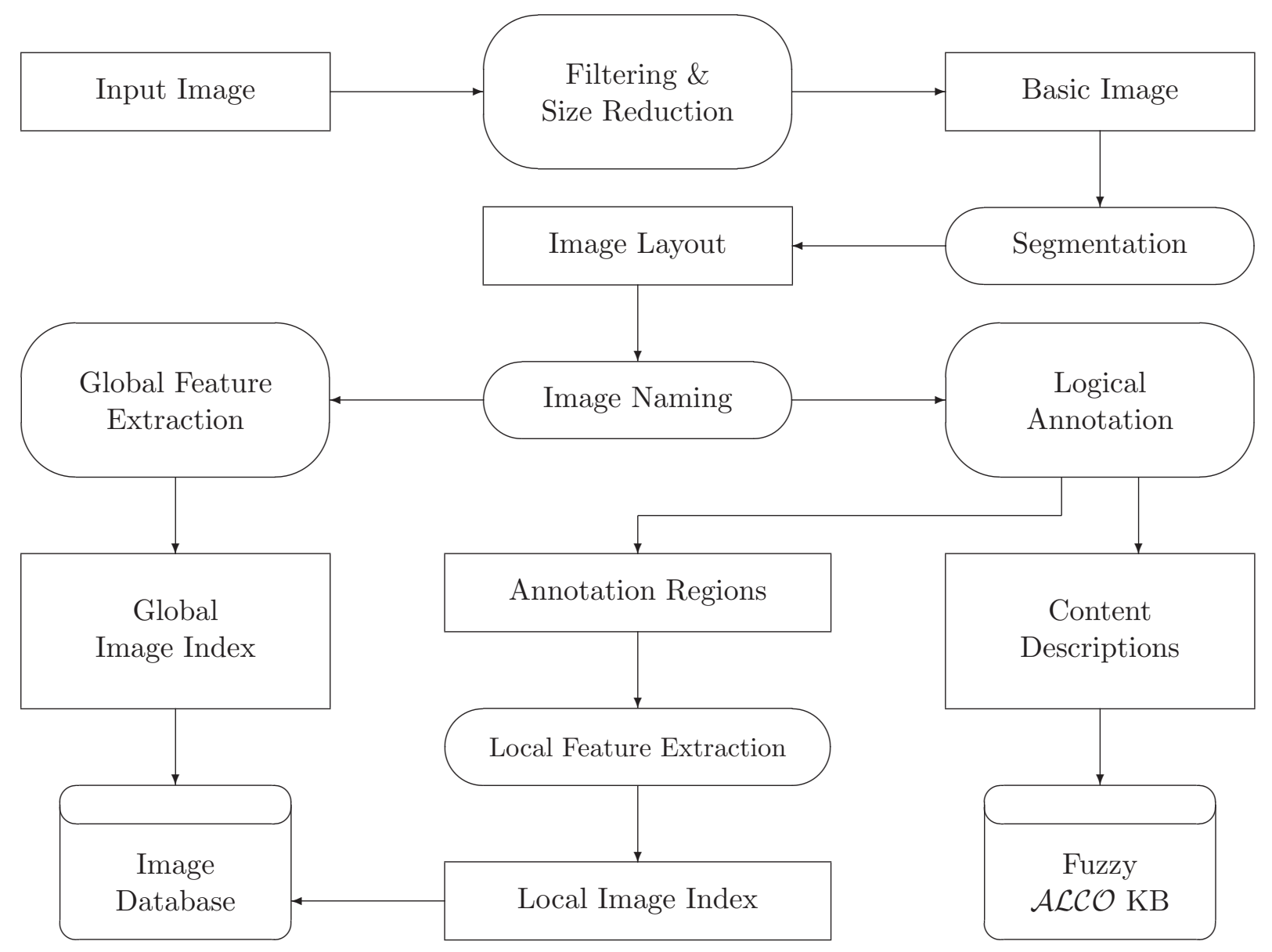

Figure 4: Image acquisition in ARIANNA
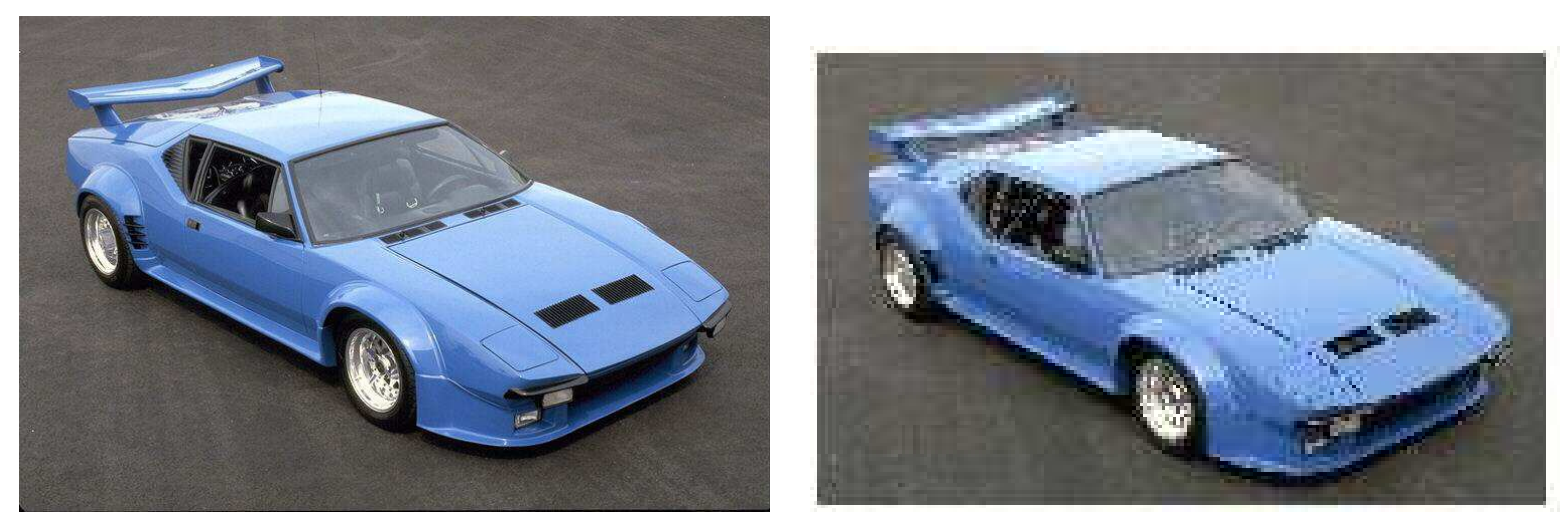

Figure 5: A Sample Input Image and the corresponding Basic Image 
Segmentation The task of the Segmentation Module is to derive the Image Layout from the Basic Image. The Image Layout is used solely to support the user in specifying the image regions that are to be annotated via Rep assertions. The derivation of the Image Layout implies two operations: segmentation and color quantization. These operations are strictly related, and in fact they are both performed by the Segmentation Module. Given the generality of the tool being presented, we have adopted a flexible solution which produces 7 different segmentations, each provided at several levels of quantization of the color space. The image indexer can use the image partition of anyone of these segmentations, or of any combination of them, in order to select the image regions to be annotated. The channels on which the Basic Image is segmented are: color, saturation, color and saturation, brightness. For each channel, 3 levels of quantizations are used, namely 3, 7, and 15 levels.

In order to obtain these segmentations, textbook techniques based on region growing have been employed; these techniques, as well as the others used for image segmentation, are not presented as not new nor central to the present context. The color, saturation, color and saturation, brightness segmentations of the sample image of Figure 5 are showed in Figure 6, in this order, from the top down (in this figure, colors are used to highlight regions and do not have direct correspondence with those in the image). In addition, two segmentations based on edges are generated, each with three levels of quantization: 2, 7 and 15 colors. Edge detection techniques have been employed to obtain these segmentations (see Figure 7). Finally, a segmentation on texture is derived, at two levels of quantization (see Figure 8). The reason for having these segmentations and not others are, of course, mostly empirical: we presume that the combination of these segmentations covers a significant range of "difficult" images.

Different presumptions would maybe lead to a different choice, but this is not important for the model. Figure 9 shows how the screen looks like after the segmentation operation has been performed on the sample image. A $3 \times 3$ grid is used to display the various images; in particular, the central image is the input image, and is surrounded by the 7 different segmentations introduced above. Only one level is shown for each segmentation, and the user can move through the different levels by clicking on the corresponding cell of the grid. The empty cell is reserved to region selection for annotation, as we will see in a moment.

Image Naming Prior to any indexing operation, the derived Image Layout must be identified as an individual of fuzzy $\mathcal{A L C O}$, and this is the objective of the Image Naming operation. When this operation is requested, the user is asked to give a name for the image being acquired; the system validates the proposed name by checking that it is not used as the name of another image. From that point on, the name becomes the unique image identifier and two operations are possible: Global Feature Extraction and Logical Annotation.

Global Feature Extraction This operation aims at deriving the representation of the image needed to answer user queries. The so obtained representation, named Global Image Index to stress its being relative to the whole image, is stored into an archive which is part of the Image Database. According to the definition of the $\Phi$ function given in Figure 3, the following features are extracted from the Basic Image named i:

- The first three moments of the image (true) color histogram for each channel $c$ of the HBS color space, that is:

$$
m_{1 c}^{\mathrm{i}^{\mathcal{I}}}=\frac{1}{N} \sum_{j=1}^{N} p_{c, j}^{\mathrm{i}^{\mathcal{I}}} \quad m_{2 c}^{\mathrm{i}^{\mathcal{I}}}=\sqrt{\frac{1}{N} \sum_{j=1}^{N}\left(p_{c, j}^{\mathrm{i}^{\mathcal{I}}}-m_{1 c}^{\mathrm{i}^{\mathcal{I}}}\right)^{2}} \quad m_{3 c}^{\mathrm{i}^{\mathcal{I}}}=\sqrt[3]{\frac{1}{N} \sum_{j=1}^{N}\left(p_{c, j}^{\mathrm{i} \mathcal{I}}-m_{1 c}^{\mathrm{i}^{\mathcal{I}}}\right)^{3}}
$$



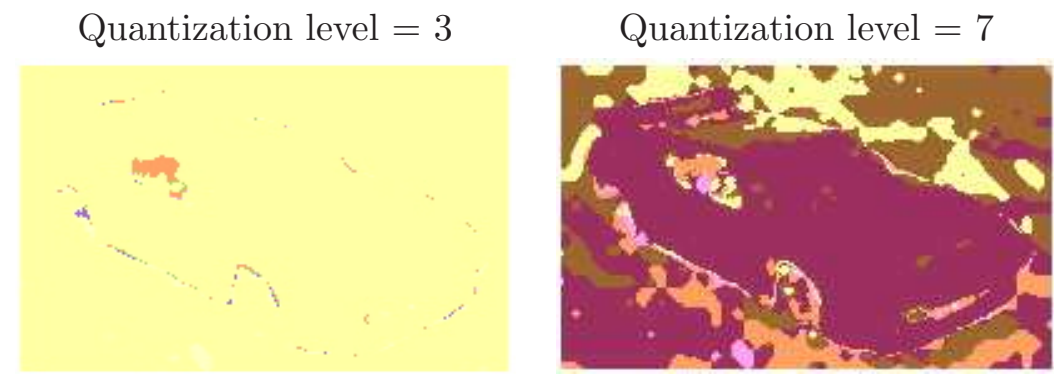

Quantization level $=15$
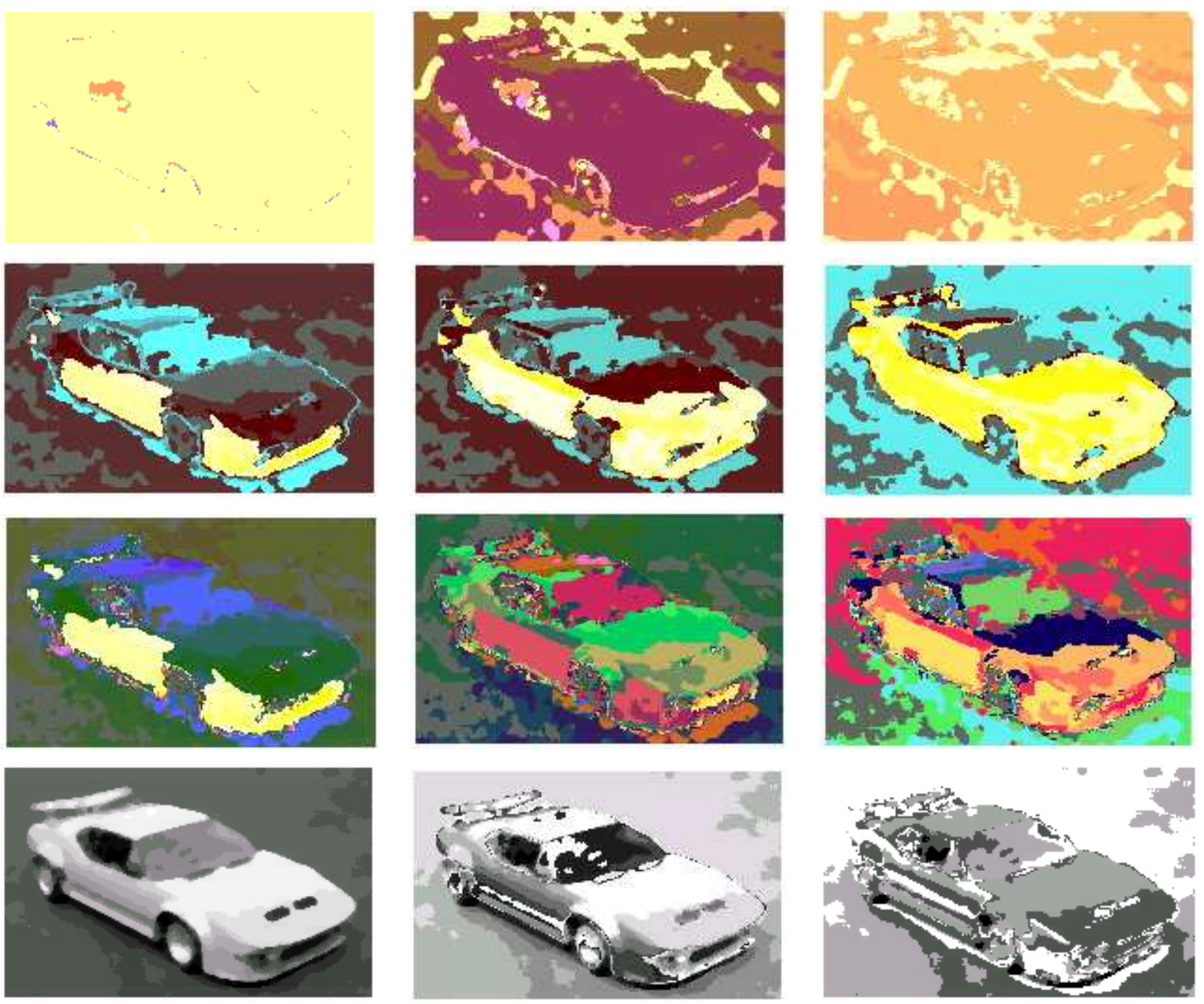

Figure 6: Segmentation by color, saturation, color and saturation, and brightness at 3 levels of quantization. 
Quantization level $=2$
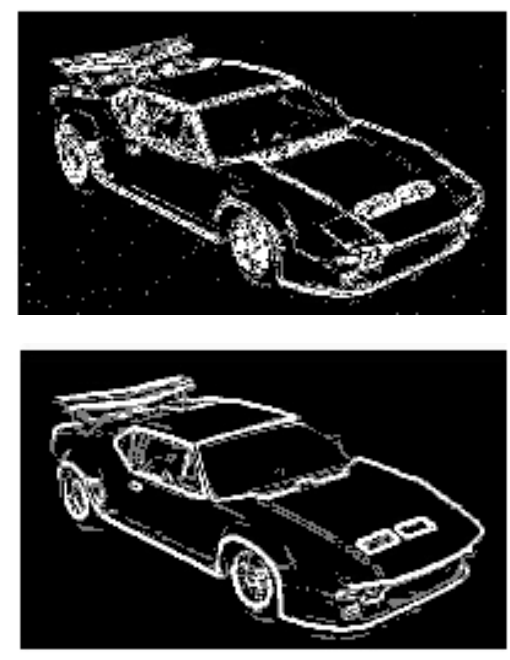

Quantization level $=7$
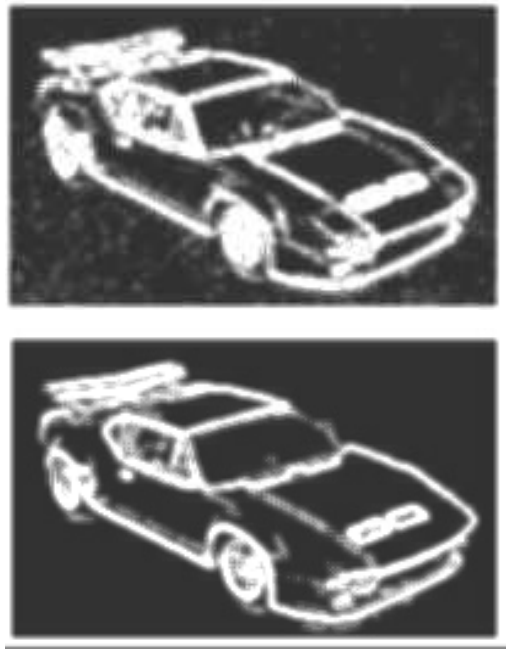

Quantization level $=15$
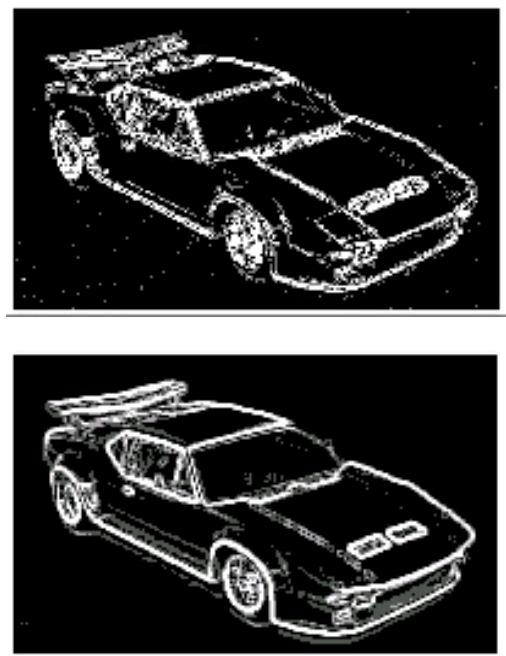

Figure 7: Segmentations by edge at 3 levels of quantization.

where $p_{c, j}^{\mathrm{i}^{\mathcal{I}}}$ is the value of the channel $c$ in the point $j$ of the image layout $\mathrm{i}^{\mathcal{I}}$. These features are extracted in order to compute a special kind of image similarity $\sigma_{i}$, namely the one based on global color [SO95]. Therefore, they will be employed upon evaluating queries of the form $(\exists \mathrm{SI} .\{\mathrm{qi}\})(\mathrm{i})$, in a way to be explained upon introducing the query module.

- The list of colors occurring in the image; this is used in order to process color queries on atomic regions, i.e. ( $\exists$ HAIR. $\exists$ HC. $\{\mathrm{c}\})(\mathrm{i})$.

- In order to evaluate queries on global color similarity (i.e. ( $\exists$ HAIR. $\exists$ HC. $\exists S C .\{c\})(i)$ ), the vector $V$ is extracted, defined as follows. $V$ has as many positions as the elements of the color set from which the user draws in specifying similar color queries on atomic regions $\left(15 \times 3 \times 3=135\right.$, in our case); the $V$ position associated to the color $\mathrm{c}^{\mathcal{I}}$ gives the degree of
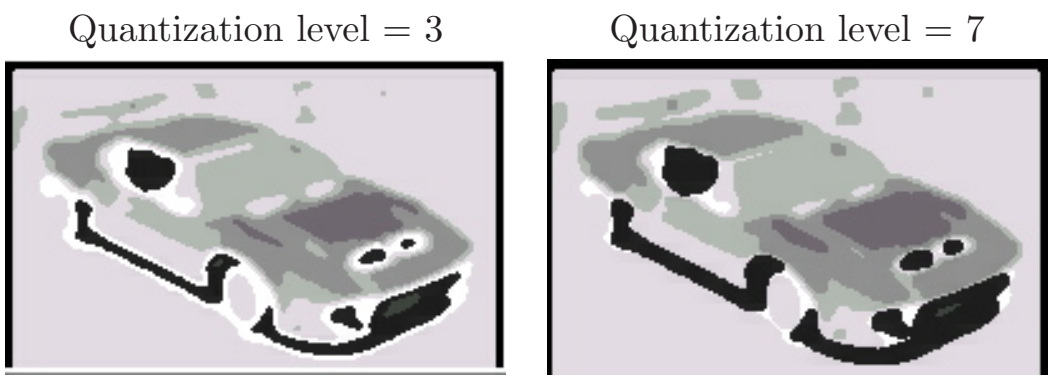

Figure 8: Segmentations by texture at 2 levels of quantization. 


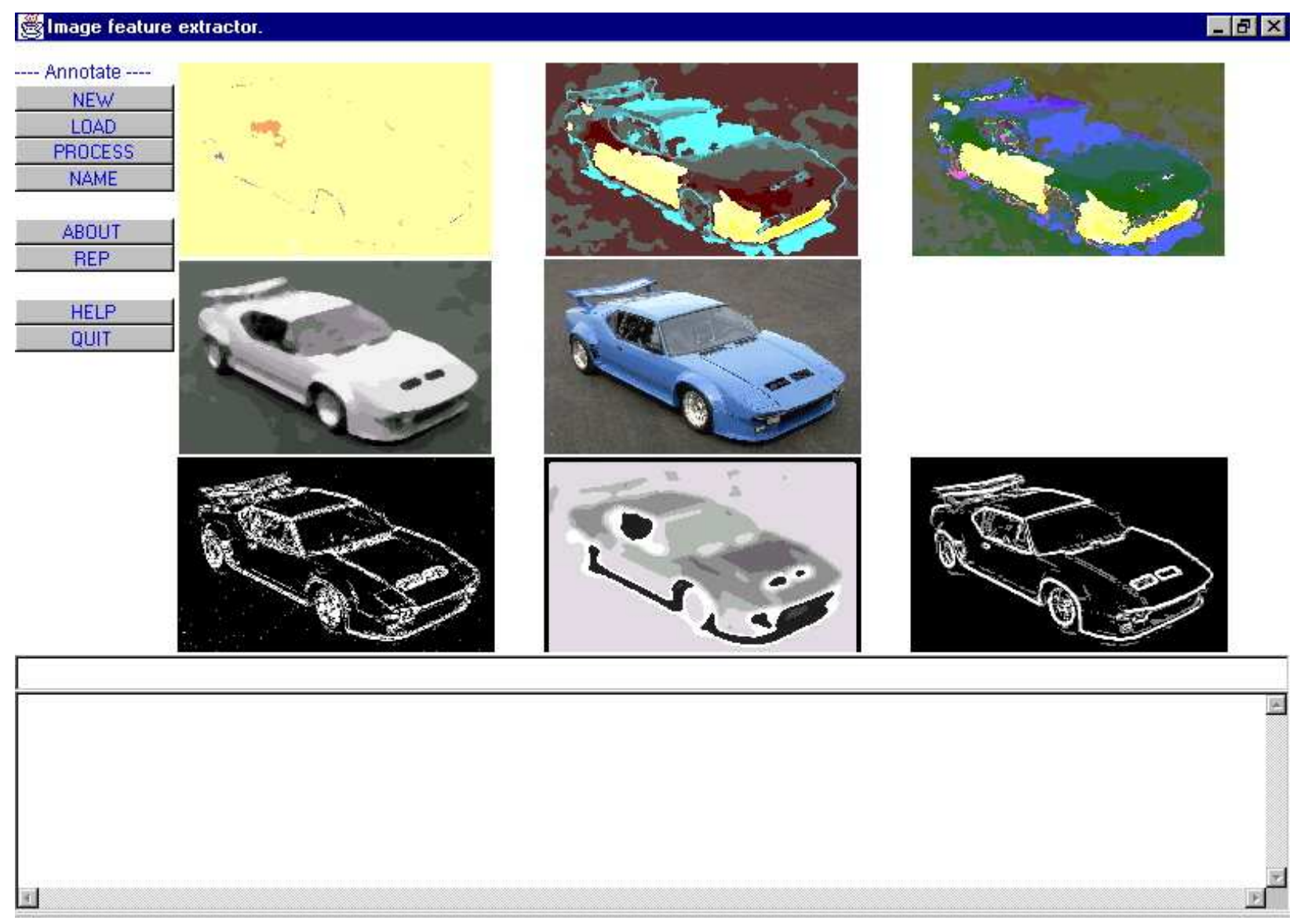

Figure 9: The screen shown by IM after segmenting the sample image. 
similarity between $\mathrm{c}^{\mathcal{I}}$ and the color in the image that best approximates it, as required by $\Phi$. The distance measure used as color similarity function $\sigma_{c}$ is:

$$
\sigma_{c}\left(i^{\mathcal{I}}, j^{\mathcal{I}}\right)=\sum_{c} \sum_{k=1}^{3}\left|m_{k c}^{\mathrm{i}^{\mathcal{I}}}-m_{k c}^{\mathrm{j}^{\mathcal{I}}}\right|
$$

where $c$ ranges, as above, on the 3 channels of the color space.

Logical Annotation This operation permits the specification of one or more Content Descriptions for named images. Upon requesting it, IM automatically creates the layout identification assertion (i.e. $\langle\operatorname{Self}(\mathrm{i}), 1\rangle$ ), and supports the creation of the other kinds of assertions. In particular:

- Situation anchoring is supported by asking the user for the name of the object to be linked to the present image via an About assertion.

- Object anchoring is supported analogously, with an additional help to the user in selecting a region image. Region naming is done "on demand", i.e. whenever a new Rep assertion is to be specified, IM automatically creates a name for the involved Annotation Region and proposes it to the indexer, who is free to use or change it. Figure 10 shows the IM screen during the selection of a region to be annotated via a Rep assertion. The region is constructed in the cell that is at the right of the cell showing the input image. The user just clicks on any region of any segmentation and, as a result, the region containing the click point is displayed. In the lower part of the screen, the identification assertion, automatically created by the system, is displayed in the format the TP expects.

The specification of situation description assertions closes the annotation of an image. Each content description is then passed to the TP, which files it in the Fuzzy $\mathcal{A L C O}$ Knowledge Base.

Local Feature Extraction Module In order to support abstract visual queries, a feature extraction task is performed on annotation regions. The extracted features make up a Local Image Index (local to annotation regions, of course), which is filed in an apposite archive of the Image Database. The structure of the local image index, relatively to the annotation region $r$, is as follows (see Figure 3):

- The name of the region $r$.

- The region color histogram, used to process color queries on the region (i.e. $(\exists \mathrm{HC} .\{\mathrm{c}\})(\mathrm{r}))$. Most of the entries in this histogram will be 0 , since $r$ is the union of a few atomic regions. Consequently, the histogram is not expected to be large.

- The vector $T$, having as many positions as the vector $V$ above, and used to evaluate similar color queries on annotation regions; the $T$ position associated to the color $\mathrm{c}^{\mathcal{I}}$, gives $n=$ $\max _{c \in \mathcal{C}} v_{c}$, as specified in Figure 3 .

- the shape of the region represented by the 8-contour and by 7 invariant moments [MKL97]. The former representation is used to process "precise" shape queries (i.e. $(\exists \mathrm{HS} .\{\mathrm{s}\})(\mathrm{r}))$ while the latter is used when the optional similarity condition is given (i.e. ( $\exists$ HS. $\exists S S .\{s\})(r))$. 


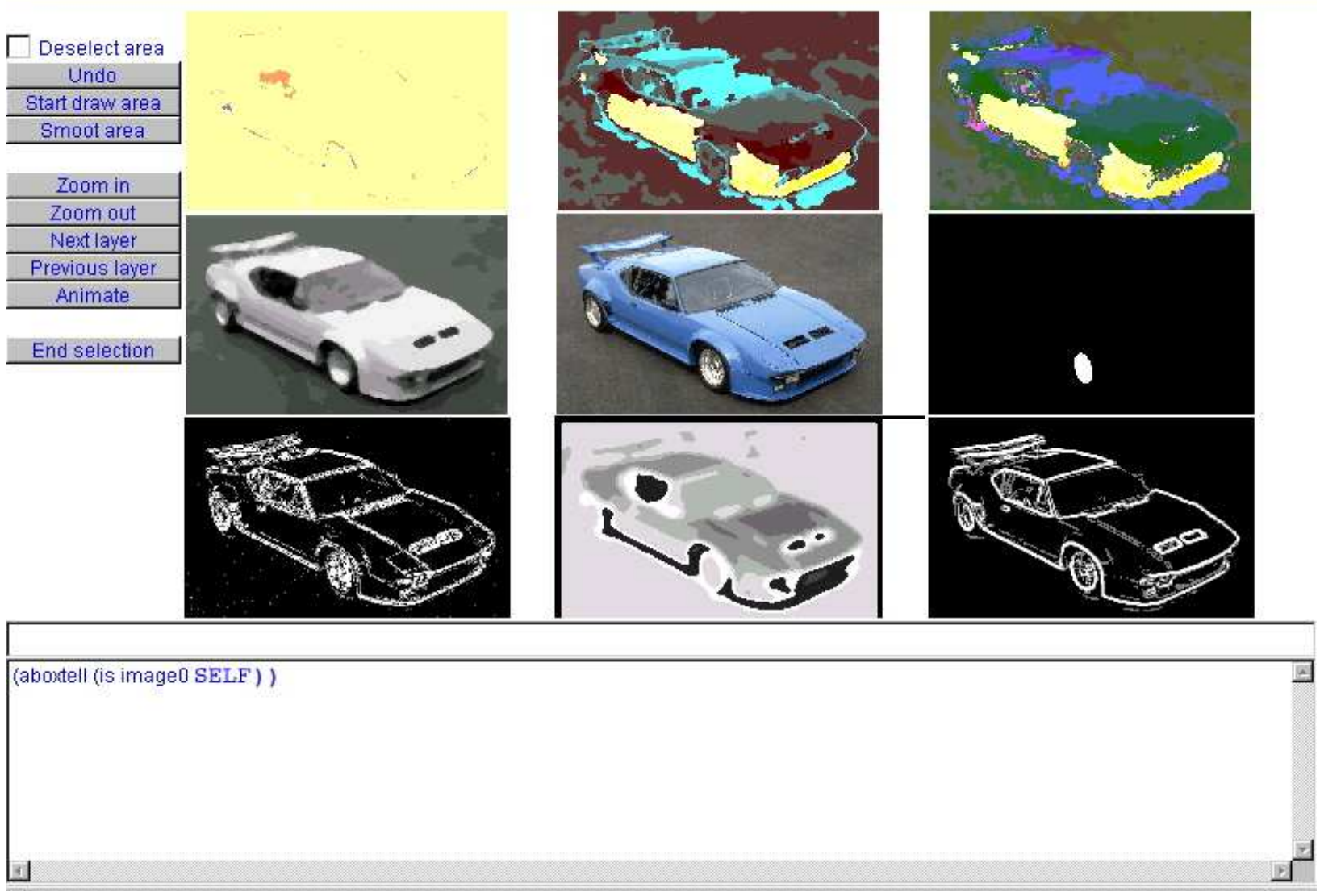

Figure 10: The screen shown by the IM during region selection.

\subsection{The query module}

The query module QM provides two basic services: query specification and query evaluation. Query specification supports the construction of image queries, performed by the user via the interface shown in Figure 11. Query evaluation is performed by the Image Query Evaluation Procedure. Given an image query $Q$, the Image Query Assertion Builder iteratively produces a query assertion $Q(i)$ for each acquired image i. The image assertion is passed on to the Image Query Decomposition $\&$ Evaluation (IQDE for short) function which implements the evaluation by decomposition process described in detail in Section 3.

In deriving $\Phi(Q(i))$ the IQDE accesses the Image Database in order to fetch the Global Image Index of $i$ and the Local Image Index of each of i's annotation regions, both built during i's acquisition. The way these representations are used by the IQDE is mostly straightforward, once one bears in mind the definition of $\Phi$ for image queries and the structure of the representations them-

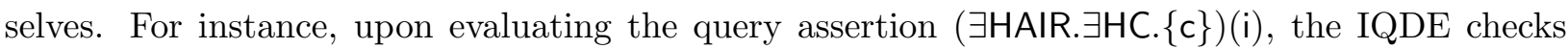
whether $\mathrm{c}$ is in the list of colors occurring in $i$, which is part of the global index; if the check is positive, then the assertion $\langle(\exists$ HAIR. $\exists$ HC. $\{c\})(i), 1\rangle$ is generated. Analogously, in order to evaluate the query $(\exists \mathrm{HIR} . C)(\mathrm{i})$, the IQDE generates an assertion $\left\langle\mathrm{HIR}\left(\mathrm{i}, \mathrm{r}_{1}\right), 1\right\rangle$ for each annotation region $r$, then applies itself to the evaluation of $C(r)$. As a final example, ( $\exists$ HC. $\exists S C .\{c\})(r)$ is evaluated by generating a fuzzy assertion whose degree of truth is the value found in the appropriate position of the $T$ vector, which is part of r's local index. 


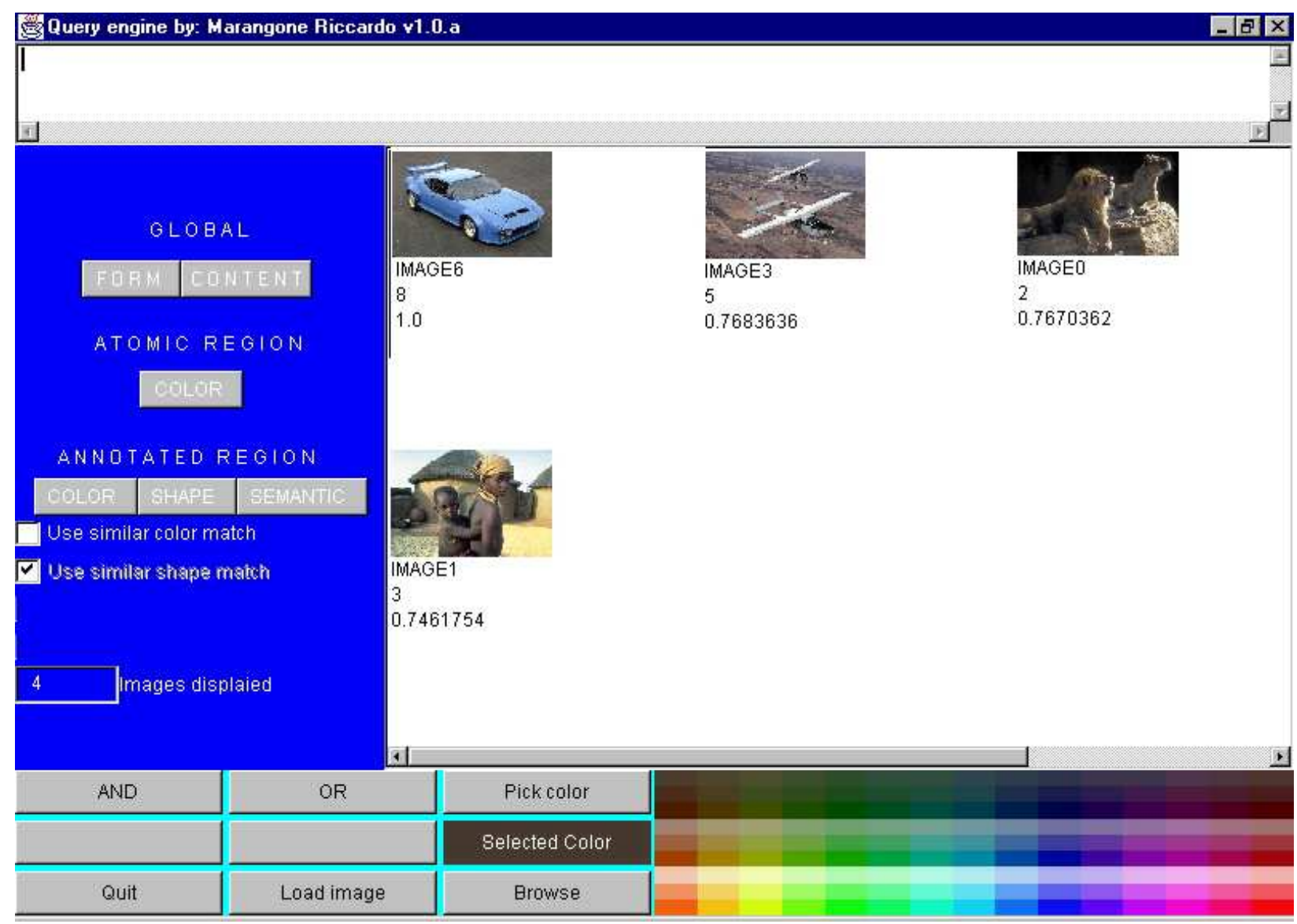

Figure 11: The QM displaying the result of a query.

The last step of the evaluation procedure is the invocation of the TP, to which the query assertion $Q(\mathrm{i})$ is sent with the purpose of computing its $m$ value against: (a) the context knowledge base $\Sigma_{D}$, (b) i's content descriptions (both these are part of the Fuzzy $\mathcal{A L C O}$ Knowledge Base maintained by the TP); and (c) the just computed $\Phi(Q(\mathrm{i}))$.

\section{Conclusions}

We have presented a system for building prototypical image retrieval applications that reconciles in a unique, well-founded framework the many functionalities that are usually found under the image retrieval label. The most important contribution of the system is the full and proper use of semantics and knowledge, while offering, at the same time, the similarity-based kind of retrieval that is undoubtedly the most significant contribution of the research carried out in these two areas during the last decade. At present, to the best of our knowledge, no other model offering the same functionalities as the one presented here, exists. Since the representations handled by the model have a clean semantics, further extensions to the model are possible. For instance, image retrieval by spatial similarity can be added: at the form level, effective spatial similarity algorithms (e.g. [GR95]) can be embedded in the model via procedural attachment, while significant spatial relationships can be included in content descriptions by drawing from the many formalisms developed within the qualitative spatial reasoning research community [Coh96]. Analogously, the model can be enhanced 
with the treatment of texture-based similarity image retrieval.

\section{References}

[Coh96] A. G. Cohn. Calculi for qualitative spatial reasoning. In Proceedings of AISMC-93, Lecture Notes in Computer Science, Steyr, AT, 1996. Springer Verlag, Heidelberg, DE.

[GR95] V. N. Gudivada and V. V. Raghavan. Design and evaluation of algorithms for image retrieval by spatial similarity. ACM Trans. on Information Systems, 13(2):115-144, 1995.

[MKL97] B. Mehtre, M. S. Kankanhalli, and W. F. Lee. Shape measures for content based image retrieval: a comparison. Information Processing and Management, 33(3):319-336, 1997.

[MSS97a] C. Meghini, F. Sebastiani, and U. Straccia. Modelling the retrieval of structured documents containing texts and images. In Proceedings of ECDL-97, n. 1324 in Lecture notes in computer science. Springer Verlag, Heidelberg, DE.

[MSS97b] C. Meghini, F. Sebastiani, and U. Straccia. The terminological image retrieval model. In Alberto Del Bimbo, editor, Proceedings of ICIAP'97, n. 1311 in Lecture notes in computer science, pages 156-163. Springer Verlag, Heidelberg, DE.

[MSS98] C. Meghini, F. Sebastiani, and U. Straccia. A model of multimedia information retrieval. Tech. Rep. B4-21, CNR-IEI, Sept. 1998. Submitted for publication.

[SO95] M.A. Stricker and M. Orengo. Similarity of color images. In Proceedings of SPIE-95, 3rd SPIE Conference on Storage and Retrieval for Still Images and Video Databases, pages 381-392, 1995.

[Str98] U. Straccia. A fuzzy description logic. In Proceedings of AAAI-98, 15th Conference of the American Association for Artificial Intelligence, pages 594-599, Madison, US, 1998. 